suggests that it is not V1, as, for one of the monkeys, the V1 information was more accurate than behavior.

Another point to consider is the curiously overspecialized form of this perceptual learning. Earlier work ${ }^{1}$ suggests that what the monkeys in this study learned is not a general ability to extract contours from noise, but the specific ability to extract this contour from this noise. The skill likely would not even generalize to the same contour rotated by 90 degrees. It is as though the brain is 'overfitting' to the new sensory input. If perceptual learning is a brain mechanism for adapting to changed sensory environments, it would be important to know how it generalizes from this overly specific learning.

Before ending, it is worth considering the reasons why Yan et al. ${ }^{2}$ found a clearer link between perceptual learning and V1 plasticity than in earlier studies. They used an electrode array, chronically implanted. They therefore observed the full spatial pattern and temporal development of neural activity, making the link to behavior unambiguous. The differential on-contour increase and off-contour decrease of activity seen here could also explain the inconsistencies in earlier fMRI studies (reviewed in ref. 1), as the differential pattern could sum to a net increase or decrease of activity at the coarse resolution of fMRI. Finally, the success of the current study could reflect the choice of taskdetecting a contour embedded in noise, rather than the orientation discrimination $\operatorname{task}^{10,11}$ used in some earlier studies. This may tie in with growing evidence that the role of V1 is not just to analyze visual scenes into oriented elements, as earlier believed, but into mid-level visual structure such as textures, contours and object boundaries ${ }^{12}$. The current stimulus was therefore likely one of the most appropriate for testing function and plasticity in V1.
This study by Yan et al. ${ }^{2}$ provides a clear result tying in with a rich set of new questions. We look forward to what lies ahead.

\section{COMPETING FINANCIAL INTERESTS}

The author declares no competing financial interests.

1. Sagi, D. Vision Res. 51, 1552-1566 (2011).

2. Yan, Y. et al. Nat. Neurosci. 17, 1380-1387 (2014)

3. Gilbert, C.D. \& Li, W. Neuron 75, 250-264 (2012).

4. Fahle, M. \& Daum, I. Neuropsychologia 40 1167-1172 (2002)

5. Dosher, B.A. \& Lu, Z.-L. Vision Res. 39, 3197-3221 (1999).

6. Li, W. \& Gilbert, C.D. J. Neurophysiol. 88, 2846-2856 (2002).

7. Li, W., Piëch, V. \& Gilbert, C.D. Neuron 50, 951-962 (2006).

8. Li, W., Piëch, V. \& Gilbert, C.D. Neuron 57, 442-451 (2008).

9. Chen, M. et al. Neuron 82, 682-694 (2014).

10. Schoups, A., Vogels, R., Qian, N. \& Orban, G.A. Nature 412, 549-553 (2001).

11. Ghose, G.M., Yang, T. \& Maunsell, J.H.R J. Neurophysiol. 87, 1867-1888 (2002).

12. Gilbert, C.D., Das, A., Ito, M., Kapadia, M.K. \& Westheimer, G. Proc. Natl. Acad. Sci. USA 93 615-622 (1996).

\title{
A two-way street
}

As any graduate student in neuroscience can attest, the hippocampus, given its importance in memory formation and its popularity as a model system for studying synaptic transmission and plasticity, has been one of the most well-studied brain regions. It has been thought to have a prescribed feedforward architecture, whereby excitatory axons originating in its CA3 subfield run along its transverse axis to form synaptic connections with CA1 pyramidal neurons, which in turn contact other excitatory neurons in the subiculum. This circuitry forces information to flow unidirectionally towards the cortex. On page 1362 of this issue, Jackson et al. challenge this notion of unidirectionality by demonstrating that hippocampal activity can flow 'in reverse', from the subiculum to CA3.

Jackson et al. studied theta oscillations, which are thought to be important for the acquisition and retrieval of memories and for the organization of internal representations of the environment. Such oscillations propagate through the hippocampal network in the form of travelling waves. Using an ex vivo preparation that preserves the entire intra-hippocampal excitatoryinhibitory network, Jackson et al. first showed that there were two distinct theta oscillators in the hippocampus. One of these oscillators was in CA3 and exerted its influence in the canonical direction (toward CA1). However, they also found a second, independent and slightly faster theta oscillator in the subiculum. Surprisingly, this oscillator was sending waves toward CA1, in the opposite direction of what was expected from the canonical circuitry of the hippocampus.

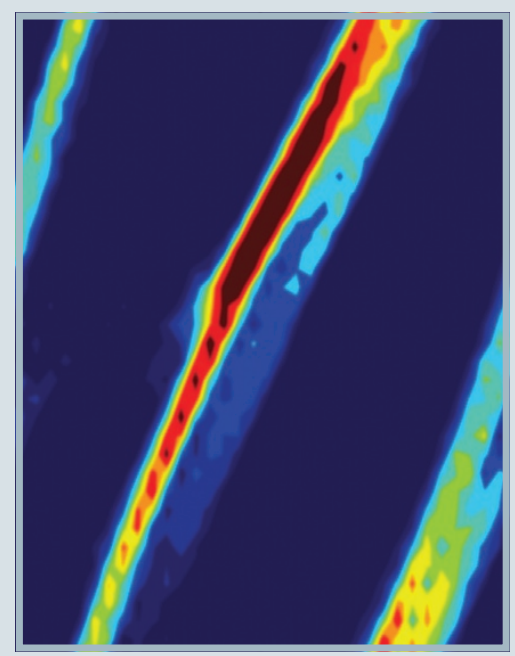
Interestingly, the two oscillators showed some phase coupling suggesting some form of interaction. The figure illustrates this phenomenon as it plots the phase coherence between CA3 ( $x$ axis) and the subiculum ( $y$ axis), with warmer colors indicating stronger phase covariations, and, in this case, a 1:2 phase locking ratio. Using an information theory-based approach, the authors showed that activity in the subiculum preceded CA3 spikes and, in a separate set of experiments, found that subicular inactivation with a sodium channel blocker changed the rhythmicity of CA3. The authors also noted that the subicular oscillator was absent in acute slices of hippocampus, which may explain why this phenomenon had not been previously reported.

What mechanisms may mediate this reverse flow of information? The subicular inactivation led to an increase in the strength of oscillatory activity in CA3, suggesting that inhibition was possibly involved. Consistent with this hypothesis, blocking $\mathrm{GABA}_{\mathrm{A}}$ receptors pharmacologically led to a decrease in the spread of activity from the subiculum to CA3 and, conversely, optogenetic activation of GABAergic neurons in the subiculum entrained CA3. The role of fast inhibition in this process suggests that long-range interneurons are involved in the backward flow of information from subiculum to CA3.

Jackson et al. went on to confirm, using in vivo recordings, that this reverse flow of information could occur in intact animals that were either exploring their environment or sleeping. Interestingly, a phase coupling similar to the one observed ex vivo was most prominent during REM sleep, suggesting that the reverse flow of information was a state-dependent phenomenon.

This study shows that CA3 and subiculum interact bidirectionally and that, counter to conventional wisdom, the hippocampus is not a 'oneway street'. Future studies will hopefully shed some light on the role that this reversed pattern of activity has in hippocampal function. 